$\Phi=\Phi$

\title{
Results and findings in the socio-economic determinants of adolescent pregnancy in Katutura, Windhoek: Namibia
}

\author{
Taimi Amakali-Nauiseb ${ }^{1}$, Susie-Ubomba Jaswa ${ }^{2}$ \\ ${ }^{1}$ School of Nursing, ${ }^{2}$ School of Public Health \\ University of Namibia, University of Limpopo \\ *Corresponding author E-mail: tnauiseb@unam.na
}

\begin{abstract}
This study focuses on the results and findings in the study on socio-economic determinants of adolescent pregnancy in Katutura, Windhoek: Namibia.

The aim of this study was to analyze and to come forth with results and findings to explore the socio-economic determinants of adolescent pregnancy in Katutura, Windhoek: Namibia.

The clinical study was a cross-sectional, descriptive study of three months duration. Semi-structured interviews methods were utilized to collect the data. A random sampling method was use to select the participant. Study population was all pregnant women attending the Katutura antenatal clinic during the time of the study. The study sample of 150 pregnant adolescent were accepted into the study. Inclusion and exclusion criteria were adhered in the selection process. Data analysis; qualitative and quantitative thematically analysis was used to interpret the data.

Based on the findings of the study $89 \%$ of the pregnant adolescent falls between the ages 17-21 who visited the ante -natal clinic. The majority interviewed represents $65 \%$ of the highest-grade completed grade $8-10.79 \%$ of the pregnant adolescents were still living with their parents. The mean age of the first intercourse was 16.5 years. $71 \%$ represent their first pregnancy, compare to $29 \%$ who said this was their second pregnancy. $46 \%$ plan to give their babies away, to their mothers which represent $84,7 \%$. $54 \%$ of the pregnant adolescent prefer to take up their parental role, responsibility and accountability compare to the $46 \%$, shifting their roles to extended families.
\end{abstract}

Keywords: Adolescence; Adoption; Contraception; Emotional Support; Financial Support.

\section{Introduction}

The article was extracted from the authors study "Socio-economic determinants of adolescent pregnancy in Katutura, Windhoek: Namibia, it focuses on the results and findings obtained. The results and findings of the study were presented that emerged from interviews conducted with pregnant adolescents in Katututra, Windhoek.

The aim of this study was to analyze and to come forth with results and findings to explore the socio-economic determinants of adolescent pregnancy in Katutura, Windhoek: Namibia.

Adolescent pregnancy is not a new phenomenon in Namibia. Few surveys have been conducted to assess this problem. Nevertheless the UNICEF Survey (HHS, 1990; NDHS, 2013) indicated that more than $50 \%$ of women have had their first child during their teens, and the mean age of this group for the first birth was 16.3 years.

As stated by Lillian and Mumbango, 2015 adolescent pregnancy is not a new issue in Namibia. There have been always young women, their partners and their family facing difficult life changing decisions because of intended or unintended pregnancy. Until recently, adolescent pregnancy was considered a private matter that involved the pregnant adolescent and the immediate family members. This issue has now however become a public concern. Namibia is no exemption to the problem of teenage pregnancy. Both the report of NDHS (2006, 2007, 2013) and USAID (2011) indicate that the rate of teenage pregnancy in Namibia stand at
$15.4 \%$ and $15 \%$ respectively. Teenage pregnancy appears unwanted because it is unplanned. Unplanned teenage pregnancy is often terminated by abortion, a negative experience that may have a lifelong emotional and social impact on her.

According to Namibia Demographic Health Survey (NDHS, 2003; NDHS, 2013) early childbearing particularly among teenagers (those under 20 years of age) have detrimental demographic, socio-economic and socio-cultural consequences. Teenage mothers are more likely to suffer from complications during delivery, which result in higher morbidity and mortality for both themselves and their children. In addition, the socio-economic advancement of teenage mothers in the areas of educational attainment and accessibility to job opportunities may be curtailed. A study conducted by Mostert (1988) found that 80 percent of the females in Katutura had their baby in their teens.

According to NDHS $(2003,2013)$ the women age $15-19$ years that are already mothers or pregnant; about one in seven adolescent women (15 percent) in Namibia are already a mother and another 3 percent were pregnant with their first child. Thus, 18 percent of adolescent women have begun the childbearing.

As indicated in the study by Omar, Hasim, Muhammad, Jaffar, Hashim and Siraj (2010) there were significant associations between adolescent pregnancy and low education level, low socioeconomic status, being raised by a single parent, not engaging in extra-curricular school activities, engaging in unsupervised activities with peers after school, and substance abuse being anemic, being unsure of the expected delivery date (Omar et al. 2010). 
In Namibia, we increasingly witness a distressing phenomenon: babies having babies. Namibia's teenage mothers are among the vulnerable groups in society. The rate of teenage pregnancy is high. According to the Demographic and Health Survey of 1993 , by age 19 , approximately $45 \%$ of all girls have already started childbearing. In the northeast the figure is as high as $75 \%$. Countrywide, $22 \%$ of the girls in the age group 15-19 were mothers (NDHS, 2003; NDHS, 2013).

According to UNICEF Report (2008), women below 20 were likely to experience maternal death four times more than women above the age of 20.Adolescent childbearing, common in many parts of the world, carries particular risks.

In Namibia the prevalence of adolescent pregnancy was $31.3 \%$; and as stated by Lillian and Mumbango 2015, adolescent pregnancy was influenced by generation, region, highest educational level, socio-economic status and cultural factors. Intervention programs and policy initiatives should focus on youth, regions, everyone regardless of the socio-economic or culture.

\section{Aim and objectives of the study}

\subsection{Aim of the study}

The aim of this study was to analyze and to come forth with results and findings to explore the socio-economic determinants of adolescent pregnancy in Katutura, Windhoek: Namibia.

In order to obtain information that would be useful in identifying the kinds of problems adolescents faced in order to design programs that will meet their needs with regard to sexual matters.

\subsection{Objectives of the study}

- To identify and highlight the contributing factors to adolescent pregnancy.

- To explore the knowledge of adolescents on safe sex practices and HIV/AIDS.

- To find out the reasons why adolescent pregnancy is on the increase.

- To establish/investigate the knowledge of adolescent on contraceptive methods.

\section{Methods}

\subsection{Study design}

The clinical study was a cross-sectional, descriptive study implemented of three months duration. The study utilized qualitative and quantitative research methods.

\subsection{Study population.}

All pregnant adolescents' women who attended the Katutura antenatal clinic during the time of the study were included.

\subsection{Sample size}

One hundred and fifty pregnant adolescent were accepted into the study.

\subsection{Inclusion and exclusion criteria}

For inclusion into the study the participants were required to:

- Be a pregnant adolescent.

- Be between the ages 12 to 21 years.

- Attend Katutura antenatal clinic.

- Give informed consent.

Participants were excluded from the study on the basis of the following:

- Not pregnant adolescent.
- Younger than 12 years.

- Older than 21 years.

\subsection{Sampling method}

A random sampling method was use to select the participant e.g. every second pregnant adolescent was interviewed.

\subsection{Data collection}

The data was collected through semi-structured interviews with a total of 150 pregnant adolescents interviewed in Katutura, Windhoek, between the ages of 12 to 21 years.

Interviews are methods utilized to maintain conversations with people and develop theoretical interpretations thereof. This method is based on a series of questions that are relevant to a topic, asked by a researcher to an informant. The informant's answers constitute the data. Interviews are used in four ways: structured, semi-structured, and focused and unstructured focused interviews (May, 1993; Brink, 2012).

Semi-structured interviews are open-ended questions that enable the researcher to gain detailed understanding of a respondent's perception of a particular topic (Smith, 1995; Brink, 2012). It is flexible as it allows the researcher to seek elaboration and clarification on important themes that emerge from an interview. The informant is also able to give a fuller understanding and perception of a topic (Smith, 1995; Brink, 2012).

The interviews were conducted on specific days, on Mondays and Thursdays, which were the follow-up days for antenatal clinic. At the beginning of the interview the researcher welcomed and thanked the participant individually for volunteering to participate. The researcher introduced her and explained the purpose of the study and interview to ensure that there was no confusion. An explanation was also given regarding the process of the interview. The researcher further explained the confidential manner in which the interview will be treated and that anonymity will be ensured, no need for identification by name during the interview. The researcher carried out the interview in the following languages, English, Afrikaans and Oshiwambo.

The structured interview methodology provided us with the opportunity and the ability to truly understand adolescents' lives and problems experienced. Although, the importance of conducting the study in both the urban and rural setting to get a clear picture The interviews has been divided into four subsection namely demographic data, background information, obstetric history and general/social data. Some questions have been taken out for illustration of the findings.

The pilot study conducted at Windhoek Central hospital, antenatal clinic and the study was carried out at Katutura State hospital, antenatal clinic.

\subsection{Permission of the study}

Prior to the start of the investigation, a protocol was submitted to the committee for research of the National School of Public Health, Medunsa, Pretoria for approval. The protocol was approved.

Application was also submitted with the Ministry of Health and Social Services in Windhoek, Namibia through the permanent secretary's office and permission was granted.

\subsection{Ethical issues}

The pregnant adolescents participated voluntarily and were free to refuse to divulge certain information about them. The direct consent for participation was obtained from pregnant adolescents themselves and privacy was assured (Brink, 2010; Brink, 2012). Anonymity was of great importance and pregnant adolescents were respected and requested not to write their names on the questionnaires (Brink, 2010; Brink, 2012). 
Pregnant adolescents assured that all information to be kept confidential. Data used for the stated purpose and no other person to have access to interview data/information (Brink, 2010; Brink, 2012).

\section{Results and discussion}

\subsection{Demographic data}

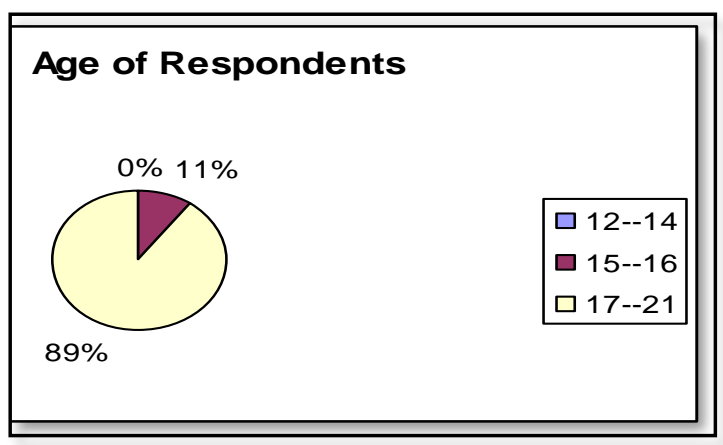

Fig. 1: Age of the Pregnant Adolescents.

The majority of the pregnant adolescents visiting the antenatal clinic were between the ages of 17-21, in the late adolescent stage $89 \%$, followed by $15-16$ years with $11 \%$ and $12-14$ years with $0 \%$.

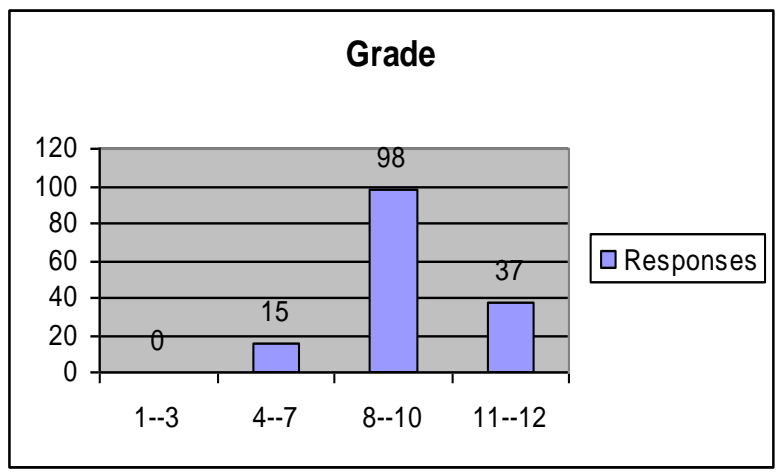

Fig. 2: Highest Grades Completed.

Out of 150 of the pregnant adolescent interviewed, 98 did complete grade $8-10(65 \%)$, meaning they were aware of pregnancy with its consequences. The total of 37 out of 150 completed grade $11-12$ (25\%), which put them in the second place, followed by those who completed grade $4-7(10 \%)$ and those who completed grade 1-3 in the last place with $0 \%$.

\subsection{Background information}

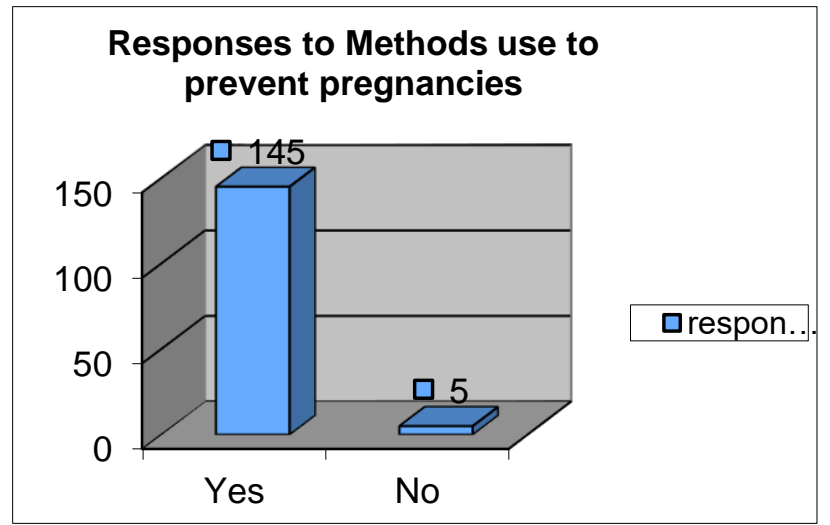

Fig. 3: Knowledge of Different Methods to Use to Prevent Pregnancy.
The majority 145 out of 150 which represents $97 \%$ said yes, they know that there were methods one can use to prevent getting pregnant. Only a small number 5 out of 150 said no, which is $3 \%$.

Table 1: First Age with Intercourse

\begin{tabular}{llllllllllll}
\hline Age & 11 & 12 & 13 & 14 & 15 & 16 & 17 & 18 & 19 & 20 & 21 \\
\hline Total & 1 & 1 & 3 & 6 & 33 & 29 & 33 & 31 & 10 & 2 & 1 \\
\hline
\end{tabular}

The above table indicated, the different age's of the pregnant adolescents when they did have their first intercourse, as well the total number of pregnant adolescents under the specific age group.

The mean age of the pregnant adolescents with their first intercourse is 16.5 years.

This correlates more or less with studies carried out by, UNICEF Survey (HHS, 1990) stating that the mean age for the first birth was 16.3, Hailonga (1993) stating that the age at first intercourse with girls is the age of 14, while the boys start at the age of 12 .

\subsection{Obstetric history}

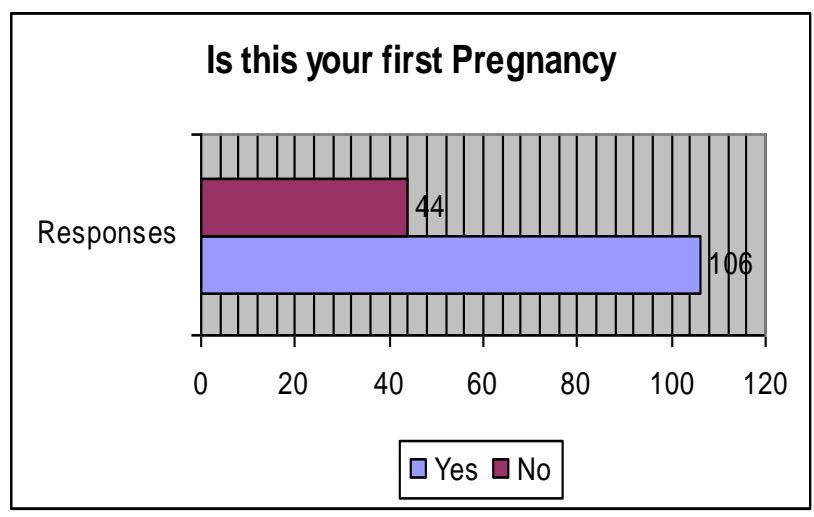

Fig. 4: First Pregnancy.

From the 150 pregnant adolescents interviewed 106 represent $71 \%$ said yes it is their first pregnancy, and 44 represent $29 \%$ said no, which implicated that this is their second pregnancy.

But the question remains are they really aware of the consequences and the risks they find themselves at this specific age being gravida 2 .

Age of current pregnancy.

The majority, which was $91 \%$, was in their third trimester (7-9 months), followed by $9 \%$ of those in their second trimester (4-6 months) and none in the first trimester (1-3 months), which was $0 \%$.

\subsection{General/social data}

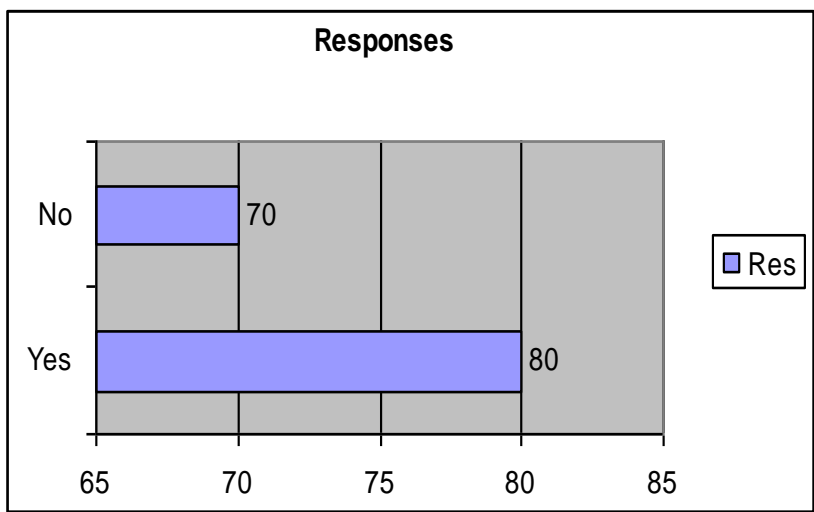

Fig. 5: Plan to Go Back to School.

The majority of the 150 pregnant adolescent, which was $53 \%$ were planning to go back to school which means to complete their secondary education (grade 12) through distance education (Namibian Center for Open Learning, NAMCOL). And those who 
were busy with tertiary education to try to complete their courses. Only $47 \%$ said no, they were not going back to school, which implies they prefer to look for a job to try to support themselves and their babies.

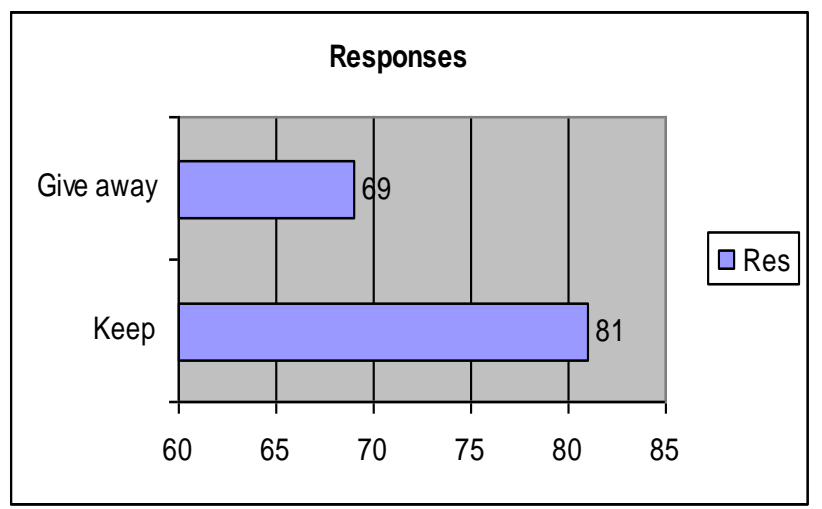

Fig. 6: Intend to Keep or Give Baby Away.

From the 150 pregnant adolescents 81 respondents, which were 54 $\%$, said they would keep this baby. While 69 respondents, representing $46 \%$ said they will give baby away. $54 \%$ of the pregnant adolescents prefer to take up their parental role, responsibility and accountability compare to the $46 \%$, shifting their roles to extended families.

\section{Recommendations}

The recommendations that emerged from the study and suggested possible future research needs. The most important theme that emerges from the discussion was that adolescents acknowledge that adolescent pregnancy is a problem and that they are at risk. Following are some of the challenges and recommendations:

\subsection{Educational programmes}

Programmes to focus on choice and responsibility in relationship to help adolescents acquire appropriate knowledge and interpersonal skills to make truly "informed choice about their sexual behaviors"

\subsection{Sex education}

The introduction of sex education as a compulsory examinable subject in school was urgently needed. If sex education was taught as a promotion subject, learners would take the information more seriously than if it were provided in voluntary classes or during extra-mural activities. It could serve as the most effective way of preventing adolescent pregnancy.

\subsection{Improved social services and health systems for youth}

Health workers should have empathy for young people as well as a capacity to listen. They must be trained to communicate effectively with young people, there is a need to project an image of trust, credibility and knowledge of adolescents and try by all means to identify with adolescent community.

\subsection{Family planning clinics for the young people}

A lot of young people don't utilize the current services because they are afraid that their parents' friends would probably oppose the use of contraceptives might be present at the clinic. By having a youth clinic they won't need to be concerned about meeting somebody's aunt or others. It is well known that unless family planning programmes receives social acceptance/legitimacy they deserve, social and psychological restraints to contraceptive usage will persist (Jagdeo, 1984).

\subsection{Parental involvement}

The situation is such that traditional beliefs discourage parents to discuss sex with their children. For this reason parents need to be involved in adolescent pregnancy prevention programmes e.g. through school committee, etc. Most importantly they should take the lead as sex educators (Hailonga, 1993).

\section{Conclusion}

In conclusion, the study examines the issue of adolescent's pregnancy from many perspectives i.e. social, economic, and psychological and many more. From the study different factors contribute to adolescent's pregnancy, financial difficulties, peer pressure, lack of parental love, and parental attitude towards sexual matters and society's attitude to the adolescent sexual activity.

The study reveals that adolescents are engaging in sexual relationships at a much younger age. They were affected by sexually related problems like childbearing, rearing and sexually transmitted disease. Moreover their sexual relations to a greater extend has an impact on their entire social relationship.

It is of importance that the Namibian society makes it their priority to act in the best interest of the adolescent; its primary consideration would be to put mechanisms in place that will allow adolescents to mature in independent adults.

Providing education to adolescent mothers serve in the long run in the best interest of the entire community.

Effective solutions to the problem depend on the following; the political will to empower women; an empathetic understanding of the situation; recognition of the interrelatedness of the underlying factors and consequences; and the adoption of holistic intervention strategies. It is of importance to adopt policies that encourage preventative strategies, than curative and rehabilitation alone.

\section{Acknowledgement}

The study was conducted with the permission of the pregnant adolescents who agreed to participate in the study at Katutura antenatal care. God be with you all!

\section{References}

[1] Brink, H. (2010). Fundamentals of Research Methodology for Health Care Professional.Cape Town, Juta \& Co. (Pty) Ltd.

[2] Brink, H. (2012). Fundamentals of Research Methodology for Health Care Professional. Cape Town, Juta \& Co. (Pty) Ltd

[3] Department of Reproductive Health and Research World Health Organization. (2011) Adolescent Pregnancy. Avenue Appia 20, CH-1211 Geneva 27, Switzerland E-mail: reproductivehealth@who.int

[4] Hailonga, P. (1993). A Study to Identify Adolescents' Knowledge, Attitudes and Beliefs towards Teenage Pregnancy. Windhoek Printers, Namibia.

[5] https://en.wikipedia.org/wiki/Windhoek.

[6] International Encyclopedia of the Social Sciences. (1968). Vol.1.Sills (Ed.). The Macmillan Company and the Free Press.

[7] Jagdeo T.P. (1984). Teenage Pregnancy in the Caribbean. International Planned Parenthood Federation, Western Hemisphere Region, New York, USA.

[8] Kozier, B. and Erb, G. (1987): Fundamentals of Nursing Concepts and Procedures. Addison-Wesley Publishing Company: California.

[9] Liang.e.l.m. (2013). Adolescent Pregnancy: A Review of the Evidence. UNFPA New York, 2013

[10] Lillian P, Mumbango T. (2015) Statistical Modeling of Adolescent Pregnancy in Namibia. J Nurs Care 4: 262. Doi: Volume 4 • Issue 4 -10.4172/2167-1168.1000262

[11] May, T. (1993). Social Research, Issues, Methods and Process, Buckingham: Open University Press. 
[12] Mensah, E. (2002). Teenage Pregnancy and its Effect on a Girl Academic Progression in Kabakaba educational circuit in the Central Region. Ghana.

[13] Minnesota Department of Health. (2010). Adolescent Pregnancy Prevention Interventions. website:http://www.advocatesforyouth.org/storage/advfy/doc uments/sspregnancies.pdf.

[14] Ministry of Education and Culture. (2008). School Policy on Learner Pregnancy in Namibia: Summary of Background Information: Gender Research \& Advocacy Project Legal Assistance Centre

[15] Ministry of Health and Social Services. (2004) Adolescent Friendly Health Services Notes. Windhoek: Namibia.

[16] Ministry of Health and Social Services. (2002) Health Information Statistics. Windhoek: Namibia.

[17] Ministry of Health and Social Services (MoHSS). (2013). Namibia Demographic Health Survey. Windhoek. Namibia.

[18] Ministry of Health and Social Services (MoHSS). (2006/7). Namibia Demographic Health Survey Windhoek. Namibia.

[19] Ministry of Health and Social Services (MoHSS). (2003). Namibia Demographic Health Survey Windhoek. Namibia.

[20] Mogotsi, I. (1998). Formal Education and Pregnancy among Learners in Namibia. The Hague: Netherlands.

[21] Mwamwenda, T.S. (1990). Educational Psychology: An African Perspective. Butterworths Professional Publishers (Pty) Ltd: Durban.

[22] Omar, K., Hasim, S., Muhammad, N. A., Jaffar, A., Hashim, S. M., \& Siraj, H. H. (2010). Adolescent Pregnancy Outcomes and Risk Factors in Malaysia. https://doi.org/10.1016/j.ijgo.2010.06.023.

[23] Pendleton, W.C. (1994). Katutura A Place Where We Stay. Gamsberg Macmillan Publishers Pty) Ltd, Windhoek: Namibia.

[24] Pendleton, W.C. (1996). Katutura: A Place Where We Stay: Life in a Post-Apartheid Township in Namibia. Gamsberg Macmillan Publishers Pty) Ltd, Windhoek: Namibia.

[25] Ryan, G.N. \& Bernard, H.R. (2000). Data Management and Analysis Methods. In Denzin, N.K. \& Lincoln, Y.S. (eds), Handbook of Qualitative Research $2^{\text {nd }}$ ed. USA: Sage Publication: 769-820.

[26] Republic of Namibia. (1995). Convention on The Elimination of all Forms of Discrimination against Women (CEDAW). First Country report. Department of Women's Affairs.

[27] Republic of Namibia. (1996). Family Planning Service Protocol for Operational Level Health Workers. Ministry of Health and Social Services. Government Printers.

[28] Republic of Namibia. (1993). Namibian Education, the Challenge of Undoing Apartheid. Ministry of Basic Education and Culture. Windhoek: Government Printers.

[29] Republic of Namibia. (1995). Policy Draft on Pregnancy among Learners in School. Ministry of Basic Education and Culture. Windhoek: Government

[30] Republic of Namibia. (1991). Proceedings of the National Safe Motherhood Conference Held in Windhoek.MOHSS, UNPF, UNICEF.Windhoek.

[31] Simon.K.(2013).Investigation of Teenage Pregnancy in Oshana Region.Namibia.

[32] Smith, J.A. (1995). Semi Structured Interviewing and Qualitative Analysis. In Smith, J.A.Horne, R and Van Langenhove, L. (Eds) Rethinking methods in psychology. London: Sage: 9-26.

[33] Saito.M.I (1998). Sex Education in School: Preventing unwanted Pregnancy in Adolescents.).December 1, 1998, Volume 63, Supplement 1, Pages S157-S160

[34] UNICEF, Namibia. (1990). Household and Nutrition Survey: MoHSS, Windhoek: Namibia.

[35] United States Agency for International Development (USAID) (2011). Teenage Pregnancy in Kavango Region: Contributing Factors and Program Recommendation.

[36] Voeten, H. (1994). Teenage Pregnancy in Namibia. Department of Cultural Anthropology University of Utrecht: Netherlands.

[37] WHO . (2014). Adolescent Pregnancy Fact Sheet, Department of Reproductive Health and Research, Geneva, Switzerland. 